\title{
Loss of inner kinetochore genes is associated with the transition to an unconventional point centromere in budding yeast
}

\author{
Nagarjun Vijay ${ }^{\text {Corresp. } 1}$ \\ ${ }^{1}$ Computational Evolutionary Genomics Lab, Department of Biological Sciences, Indian Institute of Science Education and Research Bhopal, Bhopal, \\ Madhya Pradesh, India
}

Corresponding Author: Nagarjun Vijay Email address: nagarjun@iiserb.ac.in

Background: The genomic sequences of centromeres, as well as the set of proteins that recognize and interact with centromeres, are known to quickly diverge between lineages potentially contributing to post-zygotic reproductive isolation. However, the actual sequence of events and processes involved in the divergence of the kinetochore machinery is not known. The patterns of gene loss that occur during evolution concomitant with phenotypic changes have been used to understand the timing and order of molecular changes.

Methods: I screened the high-quality genomes of twenty budding yeast species for the presence of wellstudied kinetochore genes. Based on the conserved gene order and complete genome assemblies, I identified gene loss events. Subsequently, I searched the intergenic regions to identify any un-annotated genes or gene remnants to obtain additional evidence of gene loss.

Results: My analysis identified the loss of four genes (NKP1, NKP2, CENPL/IML3, and CENPN/CHL4) of the inner kinetochore constitutive centromere-associated network (CCAN/also known as CTF19 complex in yeast) in both the Naumovozyma species for which genome assemblies are available. Surprisingly, this collective loss of four genes of the CCAN/CTF19 complex coincides with the emergence of unconventional centromeres in N.castellii and N.dairenensis. My study suggests a tentative link between the emergence of unconventional pint centromeres $\mathrm{Nd}$ the turnover of kinetochore genes in budding yeast. 
2 Loss of inner kinetochore genes is associated with the transition to an unconventional point

4

centromere in budding yeast

\section{Nagarjun Vijay ${ }^{1}$}

${ }^{1}$ Computational Evolutionary Genomics Lab, Department of Biological Sciences, Indian Institute of Science Education and Research Bhopal, Bhopal, Madhya Pradesh, India

*Correspondence: nagarjun@iiserb.ac.in

Keywords: point centromere, unconventional centromeres, kinetochore, interaction network, speciation

\section{Abstract}

Background: The genomic sequences of centromeres, as well as the set of proteins that recognize and interact with centromeres, are known to quickly diverge between lineages potentially contributing to post-zygotic reproductive isolation. However, the actual sequence of events and processes involved in the divergence of the kinetochore machinery is not known. The patterns of gene loss that occur during evolution concomitant with phenotypic changes have been used to understand the timing and order of molecular changes.

Methods: I screened the high-quality genomes of twenty budding yeast species for the presence of well-studied kinetochore genes. Based on the conserved gene order and complete genome assemblies, I identified gene loss events. Subsequently, I searched the intergenic regions to identify any un-annotated genes or gene remnants to obtain additional evidence of gene loss.

Results: My analysis identified the loss of four genes (NKP1, NKP2, CENPL/IML3, and CENPN/CHL4) of the inner kinetochore constitutive centromere-associated network (CCAN/also known as CTF19 complex in yeast) in both the Naumovozyma species for which genome assemblies are available. Surprisingly, this collective loss of four genes of the CCAN/CTF19 complex coincides with the emergence of unconventional centromeres in N.castellii and N.dairenensis. My study suggests a tentative link between the emergence of uncon tional point cen meres and the turnover of kinetochore genes in budding yeast.

The increasing availability of genomic datasets across diverse species has allowed the use of comparative genomic approaches to study kinetochore evolution. Such comparative approaches 
36 have led to interesting evolutionary insights from species with unique kinetochores (Drinnenberg 37 \& Akiyoshi, 2017). The kinetochore is a complex of interacting proteins that have undergone 38 frequent changes in gene content and exhibits strong signatures of adaptive evolution (Malik \& 39 Henikoff, 2001; Talbert, Bryson \& Henikoff, 2004; Schueler et al., 2010; Drinnenberg, Henikoff 40 \& Malik, 2016). Despite changes in gene content, the kinetochore in diverse species is known to 41 consist of the inner kinetochore which is assembled close to the centromere DNA as a 42 specialised form of histone (CENPA) and the outer kinetochore which interacts with 43 microtubules. The inner and outer kinetochores are known to consist of several interacting 44 protein complexes (see van Hooff et al., 2017 for more details). These kinetochore protein 45 complexes show patterns of co-evolution with interacting components through correlated gene 46 loss events (Tromer, 2017). The CCAN/CTF19 complex interfaces with both the inner and outer 47 kinetochore acting as a foundation for kinetochore formation (Hara \& Fukagawa, 2017; Hinshaw \& Harrison, 2019). Surprisingly, large-scale systematic screening for kinetochore genes in the genomes of numerous eukaryotes has shown that the majority of the components of the CCAN/CTF19 complex are lost in many lineages (van Hooff et al., 2017). Another unexpected observation was that the CENPA (CENH3 \& CSE4 homolog) gene, which performs a central role in kinetochore function, was lost in many insect species. This potentially recurrent loss of the CENPA/CSE4 gene in insect species coincides with their transition to holocentricity (Drinnenberg et al., 2014). Interestingly, it has been shown that C.elegans holocentromeres are actually point centromeres that are dispersed at transcription factor hotspots (Steiner \& Henikoff, 2014). Hence, it has been speculated that changes in the gene content of kinetochores might be functionally related to transitions in the centromere type (Drinnenberg \& Akiyoshi, 2017).

Centromere sequences in the vast majority of species are repeat-rich regions that are thought to be defined epigenetically and/or through recognition of dyad rich regions with non-B-form DNA structures (Kasinathan \& Henikoff, 2018). Despite advances in genome sequencing and assembly methods, the high repeat content of these centromeres makes it harder to assemble and study them. Hence, as a proxy to the study of centromere regions, comparison of tandem repeats across large phylogenetic distances have been performed to understand the evolution of centromere sequences (Melters et al., 2013). 
67 One of the most interesting transitions during centromere evolution was the emergence of 68 genetically defined point centromeres in budding yeasts (Malik \& Henikoff, 2009). These $69 \sim 150$ bp long centromeres found in Saccharomycetaceae have been easier to study due to the lack 70 of repetitive regions, availability of complete high-quality genome assemblies for multiple 71 closely related species, gene knockout libraries and ease of experimental manipulation. Hence, budding yeast species are a popular system to study the evolution of centromeres and the set of proteins that interact with them (Roy \& Sanyal, 2011).

In addition to changing their sequences, centromeres are also known to change their genomic positions without any change in the karyotype (Montefalcone et al., 1999). However, centromere evolution can also be accompanied by changes in the karyotype (O’Neill, Eldridge \& Metcalfe, 2004). Within mammals, centromeres are known to have undergone multiple repositioning events (Rocchi et al., 2012). Centromere repositioning events seem to be fairly common and could have a role in driving speciation or, at the very least, have a non-negligible role in affecting the local genomic selection landscape.

Recently, it has been shown that in Naumovozyma, an unconventional centromere has come into existence at a location that is largely distinct from that expected based on synteny with other Saccharomycetaceae species (Kobayashi et al., 2015). The suggested as a model for comparative genomics and study of adaptiv various phenotypic differences compared to other yeast species (Karader 88 2017).

In this study, I first screened the genome assemblies of eight Pre-WGD and twelve Post-WGD yeast species for the presence/absence of homologs of 67 kinetochore genes. I find evidence for the concurrent loss of multiple genes from the CCAN/CTF19 protein complex in Naumovozyma species and corresponding sequence divergence of the N-terminus region of the CENPA/CSE4 gene that interacts with the CCAN/CTF19 complex. In contrast to this, I see high levels of sequence conservation of the $\mathrm{C}$-terminus region of the CENPA/CSE4 gene that mediates an interaction between the kinetochore and centromere. My analysis finds an association between gene loss events and the emergence of novel centromeres in Naumovozyma species. 
98

99

100

101

102

103

104

105

106

107

108

109

110

111

112

113

114

115

116

117

118

119

120

121

122

123

124

125

126

127

128

129

130

\section{Materials and Methods}

\section{Gene presence/absence screening}

I compiled a list of 67 kinetochore associated genes in the yeast S. cerevisiae by downloading genes annotated with the GO term kinetochore (GO: 0000776) in Ensembl release 91. The orthologs of these genes in all twenty yeast species were identified from the Yeast Gene Order Browser (YGOB) (Byrne \& Wolfe, 2005). I screened the genomes of all twenty yeast species for the presence of all 67 genes and identified eight genes that are (both copies lost in post-WGD) lost in at least one species (see Supplementary Material S1). Kinetochore genes are known to evolve at a very fast rate, making it hard to identify orthologs of these genes even in closely related species (van Hooff et al., 2017). It is possible that these genes have evolved at a very fast rate making it unfeasible to establish homology of these genes. Fortunately, the YGOB provides not only the order of the genes but also the intergenic sequences between genes. Based on flanking genes with conserved synteny in other species, I identified the intergenic regions that correspond to the location of the missing genes (see Supplementary Material S2). I checked these intergenic sequences for the presence of open reading frames (ORFs) using the NCBI ORF finder program with default settings. The ORFs that were found in the intergenic regions did not show any homology (inferred using blastn and blastp search against NCBI's nucleotide collection and non-redundant protein sequences, respectively) to the genes that I have inferred to be lost. Based on the evidence from the identification of syntenic regions using YGOB and additional screening of intergenic regions I am confident of these gene loss events. Nonetheless, the presence of highly diverged copies of these genes in non-syntenic regions and genome assembly errors cannot be ruled out.

\section{Multiple sequence alignments of CENPA/CSE4 gene}

The complete open reading frame of the CENPA (CENH3 \& CSE4 homolog) gene was used for multiple sequence alignment. To ensure that the results I see are not the result of alignment artifacts, I performed the multiple sequence alignment at the nucleotide sequence level and amino acid residue level using four different programs (Clustal omega (default settings in the webserver), M-coffee (command used and alignment scores are provided in Supplementary Material S3), MUSCLE (default settings in MEGA) and Guidance with PRANK as the aligner 
131 (command used is provided in Supplementary Material S3)). All the nucleotide and amino acid

132 sequence alignments are provided as Supplementary Material S3. I investigated the multiple

133 sequence alignments for evidence of lineage-specific patterns of selection in Naumovozyma

134 species using the programs (RELAX, MEME, FEL and BUSTED) available in the HYPHY

135 package (Kosakovsky Pond, Frost \& Muse, 2005). The output files of these are also provided in

136 Supplementary Material S3. The presence of additional sequences in the genus Naumovozyma

137 was seen in all of the multiple sequence alignments that I have analysed. It is possible that longer

138 ORF's have been incorrectly annotated as the CDS for both Naumovozyma species. However,

139 the second methionine codon in the CDS occurs at the $226^{\text {th }}$ and $40^{\text {th }}$ residue from the currently

140 annotated start codon in N. castellii \& N. dairenensis, respectively. If the second methionine is

141 the correct start codon, the N. castellii protein would be just 39 residues. This suggests that the

142 correct ORF is annotated in Naumovozyma species. To further rule out the possibility of

143 erroneous annotation of shorter ORF's in non-naumovozyma species I extracted the genomic

144 sequence found between genes flanking CENPA (CENH3 \& CSE4 homolog) and searched them

145 for ORF's using the NCBI ORF finder program with default settings (see Supplementary

146 Material S4). I found that the longest ORF that could be identified in this sequence was the

147 currently annotated ORF itself. This further supports the validity of the annotation and multiple

148 sequence alignments generated by me. I used the amino acid alignment generated by MUSCLE

149 aligner to calculate the sequence conservation score using the al2co program (Pei \& Grishin,

150 2001) with default settings along with the -a flag to calculate nine measures of sequence

151 conservation. Per base measures of sequence conservation are provided as Supplementary

152 Material S5.

153

154 Calculation of dyad density

155 Centromere sequences of the two Naumovozyma species were obtained from NCBI and YGOB

156 browser for the remaining 18 species. The intergenic regions in Naumovozyma species

157 orthologous to the old centromeres were extracted from YGOB. All these sequences and their

158 locations are provided in Supplementary Material S6. The program palindrome from the

159 EMBOSS package (Rice, Longden \& Bleasby, 2000) was used to identify dyad symmetry (i.e.,

160 DNA sequences with base pairs that are inverted repeats of each other). The following settings

161 were used for running the program "-minpallen 5 -maxpallen 100 -gaplimit 20 -overlap". All the

Peer] reviewing PDF | (2020:03:46948:1:1:NEW 23 Aug 2020) 
162 palindromes identified for each of the sequences is provided as Supplementary Material S7.

163 The dyad density for each centromere sequence was calculated as the fraction of bases that are

164 part of a dyad. GC content for each sequence was calculated as the fraction of GC bases. GC

165 content and dyad density for each of the centromere sequences are provided in Supplementary

166 Material S8.

167 Results

168

\section{Patterns of kinetochore gene loss}

170

171

My study system consists of eight Pre-WGD and twelve Post-WGD yeast species at varying evolutionary distances (see Fig. 1, phylogeny based on (Gordon, Byrne \& Wolfe, 2011; Feng et al., 2017)). Based on screening of twenty yeast genomes for the presence of kinetochore genes I identified that eight genes (NKP1, NKP2, CENPL/IML3, CENPN/CHL4, CENPT/CNN1, CENPW/WIP1, FIN1 and BIR1) are lost at least once (see Fig. 1 and Supplementary Material S1). Intriguingly, five of the eight genes (i.e., NKP1, NKP2, CENPL/IML3, BIR1 and WIP1) that have lost both their copies in the Post-WGD species are also the ones that are lost in the Pre-

178 WGD species (see Fig. 1). This hints at the dispensability of these genes over the course of evolution. In this study, I focus on the set of four genes (NKP1, NKP2, CENPL/IML3, and CENPN/CHL4) that are lost in Naumovozyma (a genus known to have novel point centromeres). As the name suggests, the Non-essential Kinetochore Protein genes NKP1 \& NKP2 produce

182 proteins that localise to kinetochores and when deleted produce viable single gene mutants

183 (Cheeseman et al., 2002). However, both single gene mutants are known to show elevated rates

184 of chromosome loss (Fernius \& Marston, 2009). Knockout of both NKP1 and NKP2 only shows 185 a moderate increase in chromosome loss (Tirupataiah et al., 2014). Despite being non-essential 186 genes, at least one copy of both of these genes is found in 15 of the 20 yeast species screened in 187 this study. A broader phylogenetic search for homologs of these genes has shown the repeated 188 loss of these two genes in various taxa (Tromer, 2017). While it was known that NKP1 and 189 NKP2 bind to COMA complex (Hornung et al., 2014), recent work has shown that NKP1 and 190 NKP2 are positioned at the bottom of the CTF19 complex and form a four-chain helical coil 191 along with OKP1 and AME1 at their c-terminus (Hinshaw \& Harrison, 2019). The main function 192 of the NKP1 and NKP2 heterodimer is thought to be the stabilisation of the COMA complex 
193 (Schmitzberger et al., 2017). The COMA complex is known to interact directly with the

194 CENPA/CSE4 protein (Fischböck-Halwachs et al., 2019).

195

196 The CENPN/CHL4 (CHromosome Loss 4) single-gene knockouts are also viable but are known

197 to show increased levels of chromosome loss, miss-segregation, and abnormal kinetochores (Roy

198 et al., 1997). However, based on the chromatin state CENPN/CHL4 mutant cells are known to

199 show two distinct (high and low) levels of mitotic mobility (Roy \& Sanyal, 2011). This suggests

200 that CENPN/CHL4 mutants can be compensated through changes in the chromatin state.

201 CENPL/IML3 (Increased Minichromosome Loss) protein forms a stable heterodimer with

202 CENPN/CHL4 protein (Hinshaw \& Harrison, 2013). Although CENPL/IML3 and

203 CENPN/CHL4 are known interactors, the phylogenetic distribution of CENPL/IML3 seems to be

204 more restricted than that of CENPN/CHL4. The association of cohesin with the pericentromeric

205 regions is ensured by the action of CENPL/IML3 and CENPN/CHL4. The lack of these two

206 genes leads to reduced cohesin binding at the pericentromere that results in the miss-segregation

207 of chromosomes (Fernius \& Marston, 2009).

208

209 The function of the genes lost in Naumovozyma may be performed by genes that are found in

210 Naumovozyma but are absent in S. cerevisiae. Naumovozyma species have 46 genes that are

211 absent in the other 18 species analysed in this study. To evaluate whether any of these genus

212 specific genes could have taken over the role performed by the four missing genes, I identified

213 homologs of each of these genes by performing blastp search against the non-redundant protein

214 database with an e-value cut-off of $10^{-3}$. None of the identified homologs had a characterised role

215 related to the kinetochore machinery. However, profile-versus-profile search based comparisons

216 have identified that NKP1 and NKP2 are extremely similar to Mis12 and Nnf1 (Tromer et al.,

217 2019). Since, one copy (NCAS0H00450 \& NDAI0K02740) of the Nnf1 gene is present in each

218 of the Naumovozyma species; it is possible that the functions of NKP1/2 are compensated.

219

220

221 N-terminus divergence of CENPA/CSE4 gene in Naumovozyma 
223 The detailed study of the interactions between kinetochore proteins in S. cerevisiae has led to a 224 better understanding of their roles (Measday et al., 2005; Baetz, Measday \& Andrews, 2006). 225 However, the evolution of the kinetochore network across Eukaryotes has been shown to be a 226 complex process that requires further investigation (van Hooff et al., 2017). In the current study, 227 I have focussed on well-studied genetic interactions of the selected genes that have lost both 228 copies in Post-WGD yeast species. Chromatin Immuno-Precipitation (ChIP) of CENPA/CSE4 229 protein followed by sequencing of DNA fragments was used by Kobayashi et al. (2015) to 230 identify all the CENPA/CSE4 binding sites in the N. castellii genome. Based on this 231 experimental data for CENPA/CSE4 along with similar ChIP-seq data for the NDC10, NDC80, 232 and CEP3 proteins the locations of the new centromeres in N. castellii have been validated 233 (Kobayashi et al., 2015). The multiple sequence alignment of amino-acid sequences across the 234 study species (see Supplementary Material S3) shows that the CENPA/CSE4 genes of N. 235 castellii and N. dairenensis have a stretch of approximately ten amino acid residues at the very 236 beginning of the CENPA/CSE4 gene that is absent in other species. It is known that the C237 terminus of the CENPA/CSE4 protein binds to the centromeric DNA sequence, and the N238 terminus interacts with the CCAN/CTF19 complex (Chen et al., 2000). The N-terminus region 239 has overall reduced sequence conservation across all species compared to the C-terminus region 240 (see Fig. 2). I also find evidence of significant relaxed selection in the N. castellii and N. 241 dairenensis lineages using the hypothesis testing framework available in the RELAX program 242 (version 2.1) on the multiple sequence alignments generated by MUSCLE. However, when the 243 alignments generated using guidance with PRANK as aligner was used, the relaxed selection in 244 the N. castellii lineage was not found to be statistically significant (see Supplementary 245 Material S3). These differences in the inferences based on the multiple sequence aligner used 246 are known to occur when the sequences are highly diverged (Blackburne \& Whelan, 2013). I 247 have shown earlier that genes can experience relaxed selection when the c-terminal end is 248 increased in length due to change in the position of the stop codon (Shinde et al., 2019). Hence, 249 the relaxed selection detected in the CENPA/CSE4 gene could simply be a result of drastic 250 change in the length of the n-terminal sequence.

252 The N-terminus of the CENPA/CSE4 protein has been shown to play a role in the ubiquitin253 mediated proteolysis of the CENPA/CSE4 protein (Au et al., 2013). More recently, sumoylation 
254 and ubiquitination of the N-terminus have been shown to be required to prevent the 255 mislocalization of CENPA/CSE4 to non-centromeric chromatin (Ohkuni et al., 2018). The lysine 25665 residue (K65) in the CENPA/CSE4 gene has been identified as the residue important for 257 proper localisation. Changes in the N-terminus region of CENPA/CSE4 gene could have led to 258 changes in the post-translational modifications resulting in changes in the localisation patterns 259 and subsequent movement of centromeres in Naumovozyma.

260

261

262

263

264

265

266

267

268

269

270

271

272

273

274

275

276

277

278

279

280

281

282

283

284

285

\section{Dyad density at point centromeres}

Dyad symmetry refers to the presence of inverted repeats or palindromes in the sequence. The inverted repeats identified on the centromere of S. cerevisiae are provided in Fig. $\mathbf{3}$ as an example. It has been shown that centromere regions are defined by the presence of Non-B-form DNA structures resulting from the presence of dyad symmetry in the nucleotide sequence (Kasinathan \& Henikoff, 2018). While the new Naumovozyma centromeres are enriched for dyad symmetries and non-B-form DNA, the dyad symmetry was less and the SIST DNA melting and cruciform extrusion scores were lower in Naumovozyma (sensu lato, demarcated by a red box in Fig. 1) compared to sensu strictu species (demarcated by a blue box in Fig. 1 (Pulvirenti et al., 2000)). However, they utilised the contrast of sensu stricto vs sensu lato and ignore other species that have been phylogenetically placed closer to the sensu strictu species. Using a larger sample size (centromeres from various budding yeast species) I show that the GC content shows a significant negative correlation (Kendall's rank correlation coefficient tau of -0.59 , p-value < 2.2e-16) with dyad density (see Fig. 4A). This correlation is reflective of the genome-wide pattern seen in S. cerevisiae (Lisnić et al., 2005).

The unconventional centromeres in the yeast species N. castellii and N. dairenensis are in most cases located at a different genomic locus compared to the point centromeres found in other budding yeast species such as S. cerevisiae (Kobayashi et al., 2015). This movement of centromeres has partly been attributed to chromosomal re-arrangements. I show that the GC content of the intergenic regions corresponding to the older (S. cerevisiae like) centromeres is very high and dyad density is low (see Fig. 4A; red coloured circles). On the other hand the new unconventional centromeres that have been identified in Naumovozyma (see Fig. 4A; blue coloured circles) are having higher dyad densities and lower GC content. I note that the pattern is 
286 the same for both Naumovozyma species (see Fig. 4A; filled circles for N. castellii and unfilled 287 circles for N. dairenensis). The new (mean: 25.80, median: 24.77, min: $20.00 \&$ max: 35.16)

288 centromeres in N. dairenensis have a lower GC content than the older regions (mean: 31.58, 289 median: 32.90, min: 22.03 \& max: 37.32). Similarly, the new (mean: 21.36, median: 20.91, min:

29018.18 \& max: 24.55) centromeres in N. castellii have a lower GC content than the older regions

291 (mean: 30.15, median: 30.35, min: 21.82 \& max: 36.47). I used a pair-wise wilcoxon test with

292 holm multiple testing correction (also see Fig. 4B) to compare the GC content of the old and new

293 centromere regions and find a difference in both N. castellii (q-value: 0.0067 ) and N. dairenensis

294 (q-value: 0.0778). This change in dyad density and GC content probably reflects a divergence

295 that occurred after the change in the location of the centromere.

296

297

298

299

300

301

302

303

304

305

306

307

308

309

310

311

312

313

314

315

316

317

\section{Discussion}

Nucleotide sequence divergence at the centromeres themselves as well as in the coding sequence of proteins that recognise and bind to centromeres have been proposed as potential mechanisms for the build-up of post-zygotic reproductive isolation (Borodin et al., 2001). The paradoxical behaviour of centromeres to evolve rapidly while still being inherited stably makes them good candidates for loci that contribute to the process of speciation (Henikoff, Ahmad \& Malik, 2001). However, the observation that Drosophila species produce fertile offspring despite the extensive divergence of the sequence of centromere binding proteins seems to contradict this idea (Sainz et al. 2003; but see Thomae et al. 2013). Computational identification and functional characterisation of the effects of sequence divergence, loss, and duplication of the kinetochore genes in diverse species will help clarify the role of the centromere in facilitating reproductive isolation.

In this study, I provide evidence that suggests that both copies of four genes of the CCAN/CTF19 complex are lost in Naumovozyma budding yeast species that have transitioned to unconventional centromeres (Kobayashi et al., 2015). The loss of these CCAN/CTF19 complex genes is potentially mediated by changes in the N-terminus region of the CENPA/CSE4 gene (see Fig. 3B). Although I have no experimental data to suggest that the changes in the Nterminus region of the CENPA/CSE4 gene are a consequence of changes in the sequence of the centromere being bound by the $\mathrm{C}$-terminus region, it is one potential scenario that could explain 
318 the observed association between gene loss and transition to unconventional centromeres. In

319 contrast to the well-studied case of loss of CENH3 (CENPA/CSE4 homolog) gene in multiple 320 insect lineages (Drinnenberg et al., 2014) and the multiple cases of exon gain and loss (Fan et al., 321 2013), I show that transition to a new centromere sequence might be sufficient for the loss of 322 CCAN/CTF19 complex genes. Understanding the sequence of events involved in the loss of the 323 CCAN/CTF19 complex genes and the emergence of the unconventional centromere in budding 324 yeast species would help understand the loss of CCAN/CTF19 complex genes in various 325 eukaryotic lineages (van Hooff et al., 2017). The CENPA/CSE4 gene has additional sequence in 326 Naumovozyma compared to other species suggesting that the unconventional centromeres might 327 potentially be remnants of the ancestral state of point centromeres. I discuss a few other potential 328 scenarios for the sequence of events that could have led to the association observed.

329 The dispensability of the genes that are lost in Naumovozyma is supported by the viability of S. 330 cerevisiae knockouts and independent loss in the two Pre-WGD Eremothecium species. It is 331 possible that initially, these genes were sequentially lost, and the emergence of novel 332 centromeres was an adaptive response to compensate for the lost genes. Yet, the loss of three out 333 of these four genes in Eremothecium species (E. gossypii and E. cymbalariae) does not seem to 334 be associated with any noticeable changes in centromere type. The evolution of novel 335 centromeres could just be one possible solution to the loss of these genes from the CCAN/CTF19 336 complex, and Eremothecium species might have come up with a different solution. Further 337 phenotypic characterisation of Eremothecium species might shed light on this.

338

339

340

341

342

343

344

345

346 347
It has been shown that loss of RNAi leads to a shortening of the centromeres and is an important determinant of centromere evolution in fungi (Yadav et al., 2018). The N. castellii species in addition to having unconventional point centromeres, have also been shown to have a functional RNA interference pathway (Drinnenberg et al., 2009). Despite having a role in fission yeast heterochromatin specification at the centromeres, siRNA's have not been found to have any centromere-specific role in N. castellii (Kobayashi et al., 2015). Nonetheless, it would be interesting to know what role the presence of RNAi machinery in Naumovozyma might have had in the loss of CCAN/CTF19 complex genes. The Drinnenberg et al. study (2009) reports the gene expression fold change of agol knockout and dcr1 knockout in N. castellii compared to the 
348 wild type. However, none of the genes showing a fold-change greater than two are part of the

349 kinetochore gene set.

350

351 The current study does not perform any experimental characterisation of phenotypes across the

352 twenty yeast species that would be caused by knocking out the genes that I identify as lost.

353 However, kinetochore genes have been studied extensively in S. cerevisiae. Single and double

354 gene knockouts have been phenotypically characterised in great detail (Measday et al., 2005).

355 This functional data from S. cerevisiae can be extrapolated to other species by supplementing it

356 with computational predictions. Such extrapolation would, of course, have to be experimentally

357 verified by subsequent studies in the focal species. Hence, our study is merely focussed on

358 demonstrating strong patterns of associations that suggest kinetochore network rewiring in

359 Naumovozyma.

360

361 Stable separation of chromosomes into daughter cells requires proper functioning of the

362 kinetochore machinery. Reduced efficiency in such segregation of chromosomes would result in

363 reduced fertility. The high levels of gene turnover seen in kinetochore genes suggest frequent

364 changes in the interactome of kinetochore. Such frequent changes in gene content could lead to

365 differences in the interactome of closely related species. These differences in the interactome can

366 have a prominent role in mediating speciation through reproductive isolation. Reconstruction of

367 the sequence of events leading to the turnover of kinetochore machinery and centromere

368 sequence at the molecular level in budding yeast species might serve as a good test case for

369 understanding its role in speciation.

370

\section{Conclusions}

372 Rapid changes in the genomic sequence of centromeres and associated kinetochore proteins

373 between closely related species are thought to have an important role in speciation. Yet, it is not

374 conclusively known whether the centromere sequence divergence occurs first or kinetochore

375 proteins evolve to use a different centromere sequence. I look at patterns of kinetochore gene

376 loss in twenty species of yeast to identify major lineage-specific events. Interestingly, the loss of

377 four genes of the CCAN/CTF19 complex coincides with the emergence of unconventional

378 centromeres in N.castellii and N.dairenensis. I speculate that loss of these genes might have 
occurred subsequent to the divergence of the centromere sequences as their role might have been taken over by other genes. While our results cannot establish the sequence of events, the identified lineage-specific loss of kinetochores genes that are known to functionally interact serves as a molecular footprint of genetic changes that contribute to reproductive isolation between species.

\section{References}

Au WC, Dawson AR, Rawson DW, Taylor SB, Baker RE, Basrai MA. 2013. A novel role of the $\mathrm{N}$ terminus of budding yeast histone $\mathrm{H} 3$ variant $\mathrm{Cse} 4$ in ubiquitin-mediated proteolysis. Genetics 194:513-518. DOI: 10.1534/genetics.113.149898.

Baetz K, Measday V, Andrews B. 2006. Revealing hidden relationships among yeast genes involved in chromosome segregation using systematic synthetic lethal and synthetic dosage lethal screens. Cell Cycle 5:592-595. DOI: 10.4161/cc.5.6.2583.

Blackburne BP, Whelan S. 2013. Class of multiple sequence alignment algorithm affects genomic analysis. Molecular Biology and Evolution. DOI: 10.1093/molbev/mss 256 .

Borodin PM, Henikoff S, Ahmad K, Malik HS. 2001. Speciation and centromere evolution [3] (multiple letters). Science 294:2478-2480. DOI: 10.1126/science.294.5551.2478.

Byrne KP, Wolfe KH. 2005. The Yeast Gene Order Browser: Combining curated homology and syntenic context reveals gene fate in polyploid species. Genome Research 15:1456-1461. DOI: $10.1101 /$ gr.3672305.

Cheeseman IM, Anderson S, Jwa M, Green EM, Kang J seog, Yates JR, Chan CSM, Drubin DG, Barnes G. 2002. Phospho-regulation of kinetochore-microtubule attachments by the Aurora kinase Ipl1p. Cell 111:163-172. DOI: 10.1016/S0092-8674(02)00973-X.

Chen Y, Baker RE, Keith KC, Harris K, Stoler S, Fitzgerald-Hayes M. 2000. The N terminus of the centromere H3-like protein $\mathrm{Cse} 4 \mathrm{p}$ performs an essential function distinct from that of the histone fold domain. Molecular and Cellular Biology 20:7037-7048. DOI: 10.1128/mcb.20.18.7037-7048.2000.

Drinnenberg IA, Akiyoshi B. 2017. Evolutionary Lessons from Species with Unique Kinetochores. In: Centromeres and Kinetochores. 111-138. DOI: 10.1007/978-3-31958592-5 5 .

Drinnenberg IA, DeYoung D, Henikoff S, Malik HS ingh. 2014. Recurrent loss of CenH3 is associated with independent transitions to holocentricity in insects. eLife 3:e03676. DOI: 10.7554/eLife.03676.

Drinnenberg IA, Henikoff S, Malik HS. 2016. Evolutionary Turnover of Kinetochore Proteins: A Ship of Theseus? Trends in Cell Biology 26:498-510. DOI: 10.1016/j.tcb.2016.01.005.

Drinnenberg IA, Weinberg DE, Xie KT, Mower JP, Wolfe KH, Fink GR, Bartel DP. 2009. RNAi in budding yeast. Science 326:544-550. DOI: $10.1126 /$ science. 1176945.

Fan XY, Yu L, Xu HL, Li Y. 2013. Multiple intron gain and loss events occurred during the evolution of Cenp-A gene. Chinese Science Bulletin 58:2174-2178. DOI: 10.1007/s11434012-5623-z.

Feng B, Lin Y, Zhou L, Guo Y, Friedman R, Xia R, Hu F, Liu C, Tang J. 2017. Reconstructing yeasts phylogenies and ancestors from whole genome data. Scientific Reports 7:1-12. DOI: 
440

441

442

443

444

445

446

447

448

449

450

451

452

453

454

455

456

457

458

459

460

461

462

463

464

465

466

467 10.1038/s41598-017-15484-5.

Fernius J, Marston AL. 2009. Establishment of cohesion at the pericentromere by the Ctf19 kinetochore subcomplex and the replication fork-associated factor, Csm3. PLoS Genetics 5:e1000629. DOI: 10.1371/journal.pgen.1000629.

Fischböck-Halwachs J, Singh S, Potocnjak M, Hagemann G, Solis-Mezarino V, Woike S, Ghodgaonkar-Steger M, Weissmann F, Gallego LD, Rojas J, Andreani J, Köhler A, Herzog F. 2019. The COMA complex interacts with Cse4 and positions Sli15/ipl1 at the budding yeast inner kinetochore. eLife 8:e42879. DOI: 10.7554/eLife.42879.

Gordon JL, Byrne KP, Wolfe KH. 2011. Mechanisms of chromosome number evolution in yeast. PLoS Genetics 7:e1002190. DOI: 10.1371/journal.pgen.1002190.

Hara M, Fukagawa T. 2017. Critical Foundation of the Kinetochore: The Constitutive Centromere-Associated Network (CCAN). Progress in molecular and subcellular biology 56:29-57. DOI: 10.1007/978-3-319-58592-5_2.

Henikoff S, Ahmad K, Malik HS. 2001. The centromere paradox: Stable inheritance with rapidly evolving DNA. Science 293:1098-1102. DOI: 10.1126/science.1062939.

Hinshaw SM, Harrison SC. 2013. An Iml3-Chl4 Heterodimer Links the Core Centromere to Factors Required for Accurate Chromosome Segregation. Cell Reports 5:29-36. DOI: 10.1016/j.celrep.2013.08.036.

Hinshaw SM, Harrison SC. 2019. The structure of the ctf19c/ccan from budding yeast. eLife 8:e48215. DOI: $10.7554 /$ eLife.44239.

van Hooff JJ, Tromer E, van Wijk LM, Snel B, Kops GJ. 2017. Evolutionary dynamics of the kinetochore network in eukaryotes as revealed by comparative genomics. EMBO reports 18:1559-1571. DOI: 10.15252/embr.201744102.

Hornung P, Troc P, Malvezzi F, Maier M, Demianova Z, Zimniak T, Litos G, Lampert F, Schleiffer A, Brunner M, Mechtler K, Herzog F, Marlovits TC, Westermann S. 2014. A cooperative mechanism drives budding yeast kinetochore assembly downstream of CENPA. Journal of Cell Biology 206:509-524. DOI: 10.1083/jcb.201403081.

Karademir Andersson A, Cohn M. 2017. Naumovozyma castellii: an alternative model for budding yeast molecular biology. Yeast 34:95-109. DOI: 10.1002/yea.3218.

Kasinathan S, Henikoff S. 2018. Non-B-Form DNA Is Enriched at Centromeres. Molecular Biology and Evolution.

Kobayashi N, Suzuki Y, Schoenfeld LW, Müller CA, Nieduszynski C, Wolfe KH, Tanaka TU. 2015. Discovery of an Unconventional Centromere in Budding Yeast Redefines Evolution of Point Centromeres. Current Biology 25:2026-2033. DOI: 10.1016/j.cub.2015.06.023.

Kosakovsky Pond SL, Frost SDW, Muse S V. 2005. HyPhy: Hypothesis testing using phylogenies. Bioinformatics. DOI: 10.1093/bioinformatics/bti079.

Lisnić B, Svetec IK, Šarić H, Nikolić I, Zgaga Z. 2005. Palindrome content of the yeast Saccharomyces cerevisiae genome. Current Genetics 47:289-297. DOI: 10.1007/s00294005-0573-5.

Malik HS, Henikoff S. 2001. Adaptive evolution of Cid, a centromere-specific histone in Drosophila. Genetics 157:1293-1298.

Malik HS, Henikoff S. 2009. Major Evolutionary Transitions in Centromere Complexity. Cell 138:1067-1082. DOI: 10.1016/j.cell.2009.08.036.

Measday V, Baetz K, Guzzo J, Yuen K, Kwok T, Sheikh B, Ding H, Ueta R, Hoac T, Cheng B, Pot I, Tong A, Yamaguchi-Iwai Y, Boone C, Hieter P, Andrews B. 2005. Systematic yeast synthetic lethal and synthetic dosage lethal screens identify genes required for chromosome

PeerJ reviewing PDF | (2020:03:46948:1:1:NEW 23 Aug 2020) 
468

segregation. Proceedings of the National Academy of Sciences 102:13956-13961. DOI: 10.1073/pnas.0503504102.

Melters DP, Bradnam KR, Young HA, Telis N, May MR, Ruby J, Sebra R, Peluso P, Eid J, Rank D, Garcia J, DeRisi JL, Smith T, Tobias C, Ross-Ibarra J, Korf I, Chan SW. 2013. Comparative analysis of tandem repeats from hundreds of species reveals unique insights into centromere evolution. Genome Biology 14:R10. DOI: 10.1186/gb-2013-14-1-r10.

Montefalcone G, Tempesta S, Rocchi M, Archidiacono N. 1999. Centromere repositioning. Genome Research 9:1184-1188. DOI: 10.1101/gr.9.12.1184.

O’Neill RJ, Eldridge MDB, Metcalfe CJ. 2004. Centromere dynamics and chromosome evolution in marsupials. In: Journal of Heredity. 375-381. DOI: 10.1093/jhered/esh063.

Ohkuni K, Levy-Myers R, Warren J, Au W-C, Takahashi Y, Baker RE, Basrai MA. 2018. Nterminal Sumoylation of Centromeric Histone H3 Variant Cse4 Regulates Its Proteolysis To Prevent Mislocalization to Non-centromeric Chromatin. G3 (Bethesda, Md.) 8:1215-1223. DOI: $10.1534 / \mathrm{g} 3.117 .300419$.

Pei J, Grishin N V. 2001. AL2CO: Calculation of positional conservation in a protein sequence alignment. Bioinformatics 17:700-712. DOI: 10.1093/bioinformatics/17.8.700.

Pulvirenti A, Nguyen H-V, Caggia C, Giudici P, Rainieri S, Zambonelli C. 2000. Saccharomyces uvarum, a proper species within Saccharomyces sensu stricto. FEMS Microbiology Letters 192:191-196. DOI: 10.1111/j.1574-6968.2000.tb09381.x.

Rice P, Longden L, Bleasby A. 2000. EMBOSS: The European Molecular Biology Open Software Suite. Trends in Genetics 16:276-277.

Rocchi M, Archidiacono N, Schempp W, Capozzi O, Stanyon R. 2012. Centromere repositioning in mammals. Heredity 108:59-67. DOI: 10.1038/hdy.2011.101.

Roy N, Poddar A, Lohia A, Sinha P. 1997. The mcm17 mutation of yeast shows a size dependent segregational defect of a mini-chromosome. Current Genetics 32:182-189. DOI: $10.1007 / \mathrm{s} 002940050264$.

Roy B, Sanyal K. 2011. Diversity in requirement of genetic and epigenetic factors for centromere function in fungi. Eukaryotic Cell 10:1384-1395. DOI: 10.1128/EC.05165-11.

Sainz A, Wilder JA, Wolf M, Hollocher H. 2003. Drosophila melanogaster and D. simulans rescue strains produce fit offspring, despite divergent centromere-specific histone alleles. Heredity 91:28-35. DOI: 10.1038/sj.hdy.6800275.

Schmitzberger F, Richter MM, Gordiyenko Y, Robinson C V, Dadlez M, Westermann S. 2017. Molecular basis for inner kinetochore configuration through $<\operatorname{scp}>\mathrm{RWD}</$ scp $>$ domainpeptide interactions. The EMBO Journal 36:3458-3482. DOI: 10.15252/embj.201796636.

Schueler MG, Swanson W, Thomas PJ, Green ED. 2010. Adaptive evolution of foundation kinetochore proteins in primates. Molecular Biology and Evolution 27:1585-1597. DOI: 10.1093/molbev/msq043.

Shinde SS, Teekas L, Sharma S, Vijay N. 2019. Signatures of Relaxed Selection in the CYP8B1 Gene of Birds and Mammals. Journal of Molecular Evolution 87:209-220. DOI: https://doi.org/10.1007/s00239-019-09903-6.

Steiner FA, Henikoff S. 2014. Holocentromeres are dispersed point centromeres localized at transcription factor hotspots. eLife 2014:e02025. DOI: 10.7554/eLife.02025.001.

Talbert PB, Bryson TD, Henikoff S. 2004. Adaptive evolution of centromere proteins in plants and animals. Journal of biology 3:18. DOI: 10.1186/jbiol11.

Thomae AW, Schade GOM, Padeken J, Borath M, Vetter I, Kremmer E, Heun P, Imhof A. 2013. A Pair of Centromeric Proteins Mediates Reproductive Isolation in Drosophila Species. 
514

515

516

517

518

519

520

521

522

523

524

525

526

527

528

529

530

531

532

533

534

535

536

537

538

539

540

541

542

543

544

545

546

547

548

549

Developmental Cell 27:412-424. DOI: 10.1016/j.devcel.2013.10.001.

Tirupataiah S, Jamir I, Srividya I, Mishra K. 2014. Yeast Nkp2 is required for accurate chromosome segregation and interacts with several components of the central kinetochore. Molecular Biology Reports 41:787-797. DOI: 10.1007/s11033-013-2918-3.

Tromer EC. 2017. Evolution of the Kinetochore Network in Eukaryotes.

Tromer EC, van Hooff JJE, Kops GJPL, Snel B. 2019. Mosaic origin of the eukaryotic kinetochore. Proceedings of the National Academy of Sciences of the United States of America 116:12873-12882. DOI: 10.1073/pnas.1821945116.

Yadav V, Sun S, Billmyre RB, Thimmappa BC, Shea T, Lintner R, Bakkeren G, Cuomo CA, Heitman J, Sanyal K. 2018. RNAi is a critical determinant of centromere evolution in closely related fungi. Proceedings of the National Academy of Sciences 115:3108-3113. DOI: $10.1073 /$ pnas. 1713725115.

\section{Figure legends}

Figure 1: Observed loss of kinetochore genes in budding yeast species. Gene loss events have been mapped onto the widely accepted tree topology obtained from (Gordon, Byrne \& Wolfe, 2011). The whole genome duplication event separating the Pre-WGD and PostWGD species is denoted by a star. Point centromeres are common to all twenty budding yeast species and their emergence is depicted by a dark blue dot. Emergence of unconventional point centromere in Naumovozyma is depicted by a light blue dot. Sensu strictu yeast species are demarcated by a blue box and sensu lato species with neocentromeres are demarcated by a red box.

Figure 2: Conserved c-terminus of CENPA/CSE4 contrasts with the diverse n-terminus. Sequence conservation (unweighted entropy measure) along the length of the CENPA/CSE4 gene calculated using the al2co program is shown for each amino acid position. While the c-terminus is highly conserved, the n-terminus has very little sequence conservation. Refer to Supplementary Material 3 for actual multiple sequence alignments.

Figure 3: Dyads identified in the S. cerevisiae centromere CEN1. Inverted repeats were identified by the program palindrome from the emboss package. Each of the lines has the $118 \mathrm{bp}$ sequence of CEN1 and the dyad region is highlighted in red colour. The last line in the figure shows the regions (coloured green) that are covered by at least one dyad. Dyad density is calculated as the fraction of bases that are covered by a dyad.

Figure 4: Dyad density and GC content at the centromere regions. (A) Negative correlation 
550

551

552

553

554

555

556

557

558

559

560

561

562

563

\section{between GC content and dyad density at the point centromeres across budding yeast} species. The filled circles represent N. castellii and unfilled circles show N. dairenensis. Red colour is used for the intergenic regions corresponding to the older (S. cerevisiae like) centromeres. Blue colour is used for the new unconventional centromeres that have been identified in Naumovozyma. Centromeres of the remaining budding species have the * shape and are coloured black. (B) Boxplots comparing the GC content of old and new centromeres in $\mathbf{N}$. castelliiand $\mathbf{N}$. dairenensis. The new (mean: 25.80, median: 24.77, min: 20.00 \& max: 35.16) centromeres in N. dairenensis have a lower GC content than the older regions (mean: 31.58, median: 32.90, min: $22.03 \&$ max: 37.32). Similarly, the new (mean: 21.36, median: 20.91, min: 18.18 \& max: 24.55) centromeres in N. castellii have a lower GC content than the older regions (mean: 30.15, median: 30.35, min: $21.82 \&$ max: 36.47). Pair-wise Wilcoxon test is used to compare the GC content of the old and new centromeres. The q-values are obtained based on holm multiple testing correction. 
Figure 1

Observed loss of kinetochore genes in budding yeast species.

Gene loss events have been mapped onto the widely accepted tree topology obtained from (Gordon, Byrne \& Wolfe, 2011) . The whole genome duplication event separating the PreWGD and Post-WGD species is denoted by a star. Point centromeres are common to all twenty budding yeast species and their emergence is depicted by a dark blue dot.

Emergence of unconventional point centromere in Naumovozyma is depicted by a light blue dot. Sensu strictu yeast species are demarcated by a blue box and sensu lato species with neo-centromeres are demarcated by a red box.

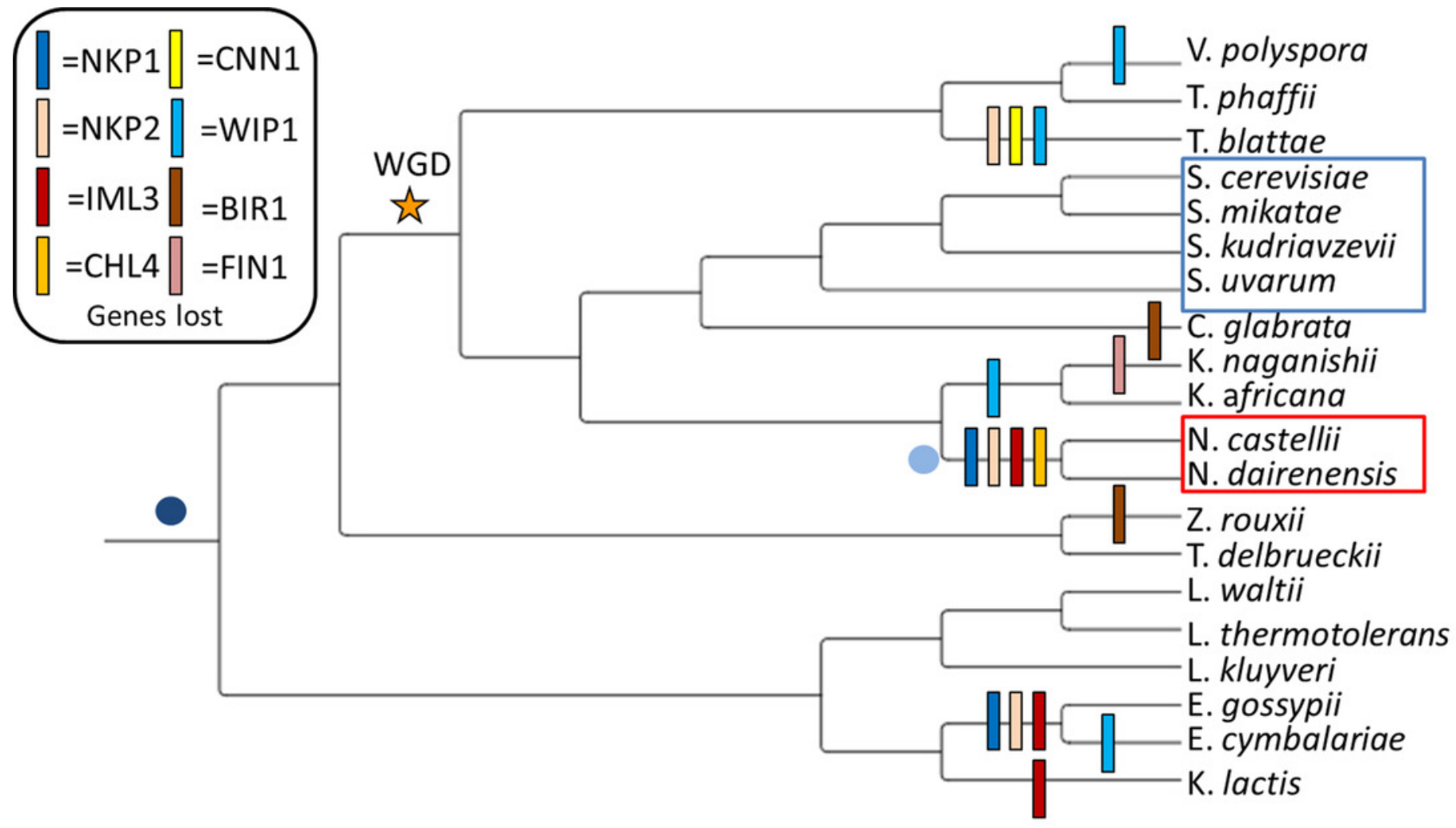


Figure 2

Conserved c-terminus of CENPA/CSE4 contrasts with the diverse n-terminus

Sequence conservation (unweighted entropy measure) along the length of the CENPA/CSE4 gene calculated using the al2co program is shown for each amino acid position. While the cterminus is highly conserved, the n-terminus has very little sequence conservation. Refer to Supplementary Material $\mathbf{3}$ for actual multiple sequence alignments.

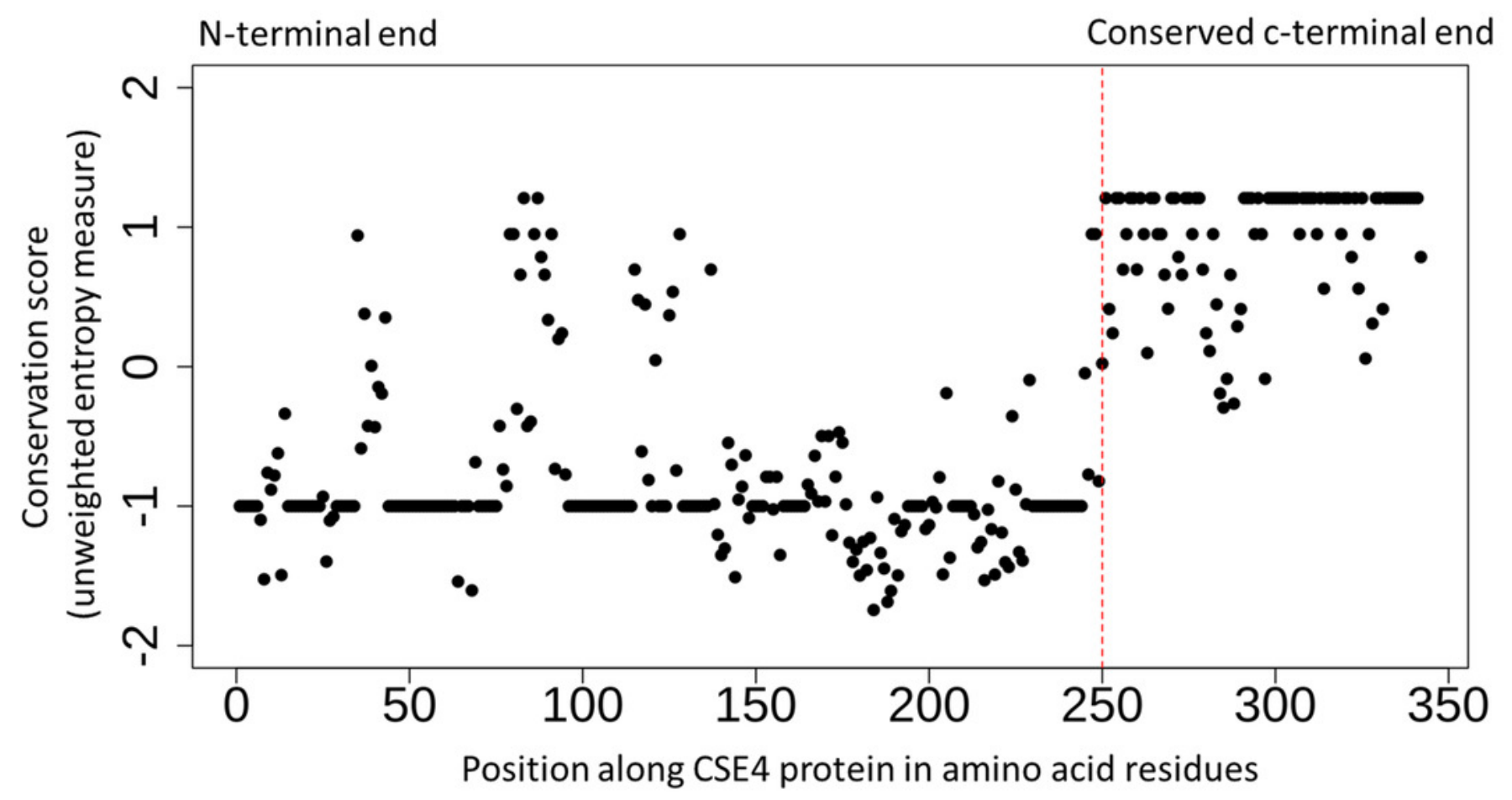




\section{Figure 3}

\section{Dyads identified in the S. cerevisiae centromere CEN1}

Inverted repeats were identified by the program palindrome from the emboss package. Each of the lines has the $118 \mathrm{bp}$ sequence of CEN1 and the dyad region is highlighted in red colour. The last line in the figure shows the regions (coloured green) that are covered by at least one dyad. Dyad density is calculated as the fraction of bases that are covered by a dyad.

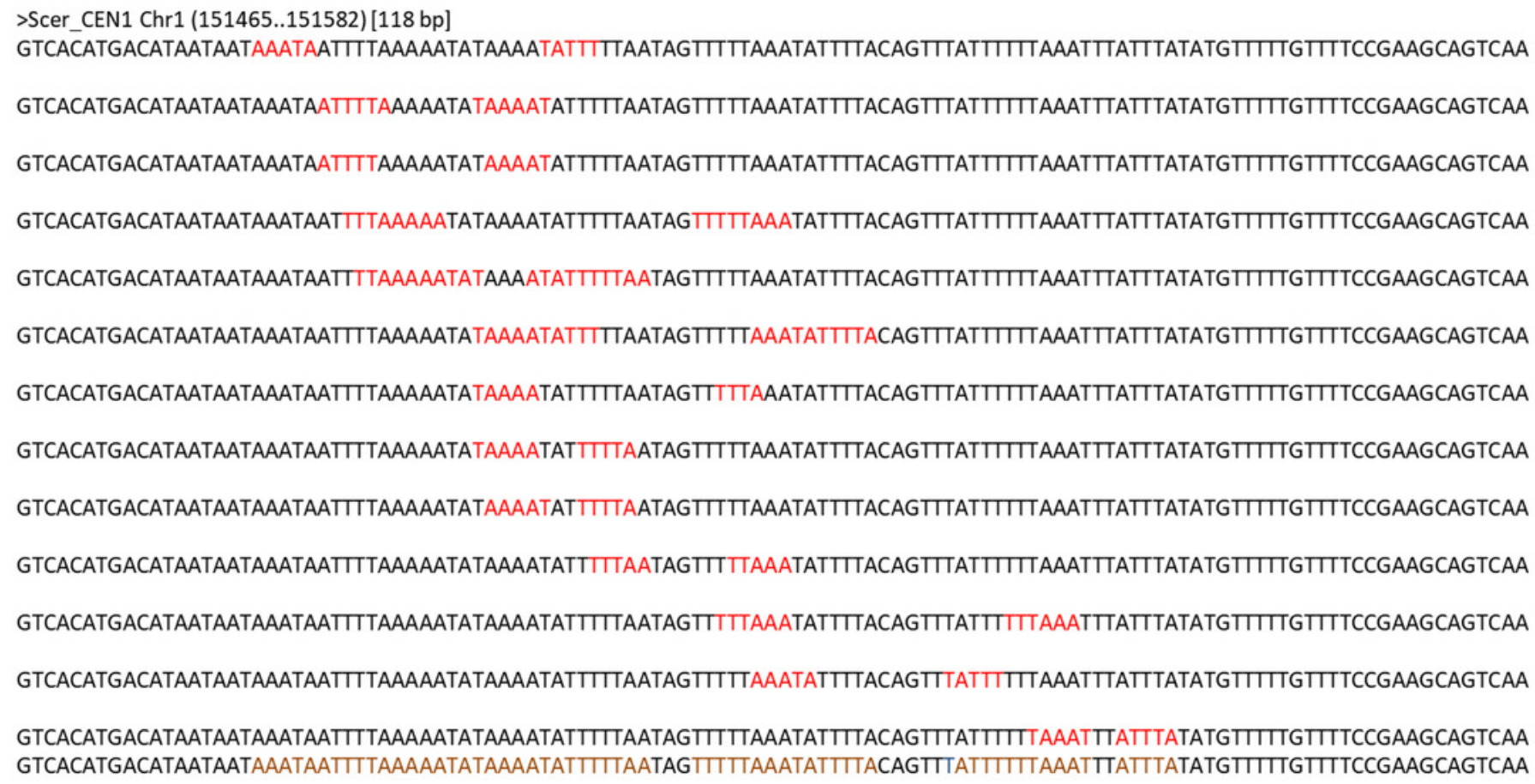




\section{Figure 4}

Dyad density and GC content at the centromere regions.

\section{(A) Negative correlation between GC content and dyad density at the point}

centromeres across budding yeast species. The filled circles represent $\mathrm{N}$. castellii and unfilled circles show N. dairenensis. Red colour is used for the intergenic regions corresponding to the older (S. cerevisiae like) centromeres. Blue colour is used for the new unconventional centromeres that have been identified in Naumovozyma. Centromeres of the remaining budding species have the * shape and are coloured black. (B) Boxplots comparing the GC content of old and new centromeres in $\mathbf{N}$. castellii and $\mathbf{N}$. dairenensis. The new (mean: 25.80, median: 24.77, min: $20.00 \& \max$ : 35.16) centromeres in N. dairenensis have a lower GC content than the older regions (mean: 31.58, median: 32.90, min: $22.03 \&$ max: 37.32). Similarly, the new (mean: 21.36, median: 20.91, min: 18.18 \& max: 24.55) centromeres in N. castellii have a lower GC content than the older regions (mean: 30.15, median: 30.35, min: $21.82 \& \max : 36.47$ ). Pair-wise Wilcoxon test is used to compare the GC content of the old and new centromeres. The q-values are obtained based on holm multiple testing correction. 
(A)

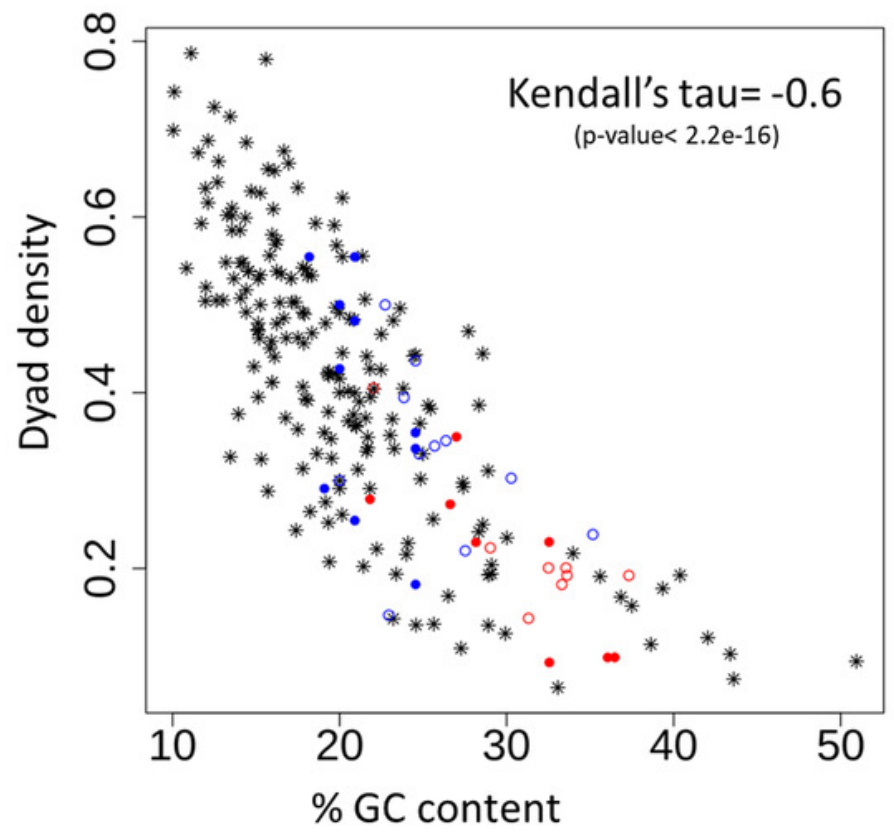

(B)

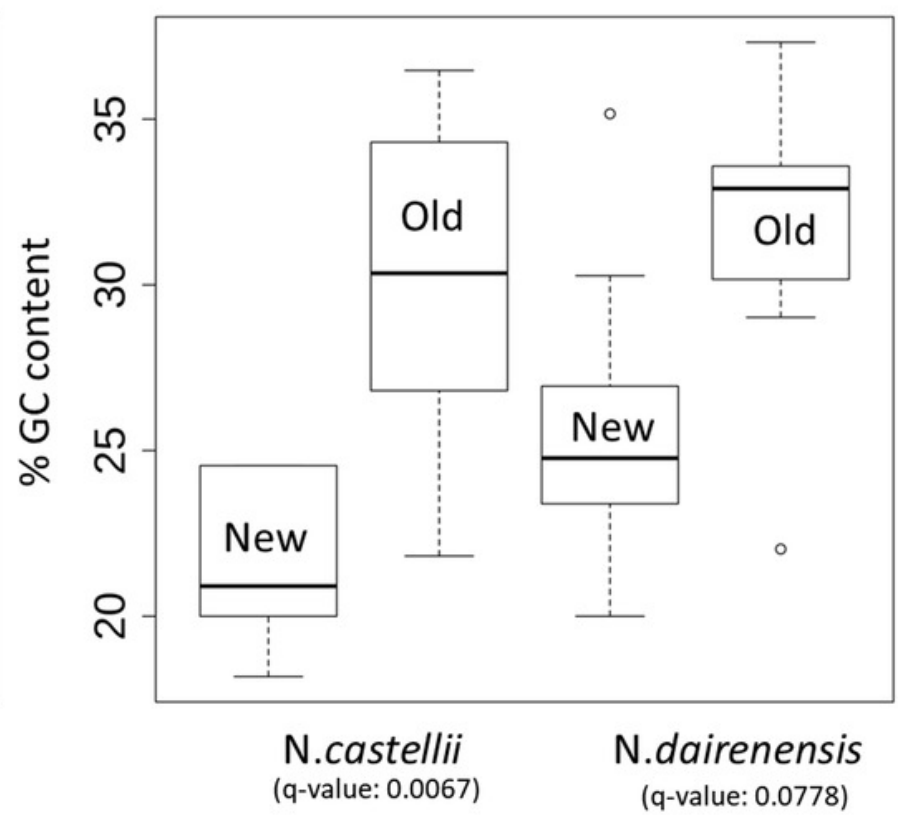

